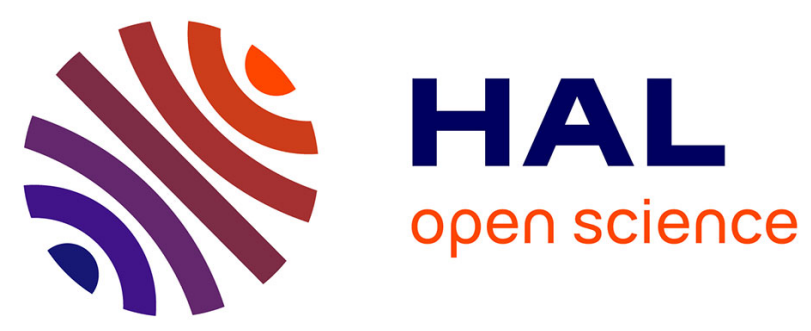

\title{
Simple modeling of self-oscillations in nanoelectromechanical systems
}

Arnaud Lazarus, T. Barois, S. Perisanu, P. Poncharal, Paul Manneville, Emmanuel de Langre, S.T. Purcell, P. Vincent, Anthony Ayari

\section{To cite this version:}

Arnaud Lazarus, T. Barois, S. Perisanu, P. Poncharal, Paul Manneville, et al.. Simple modeling of self-oscillations in nanoelectromechanical systems. Applied Physics Letters, 2010, 96 (19), pp.193114. 10.1063/1.3396191 . hal-01021117

\section{HAL Id: hal-01021117 \\ https://hal-polytechnique.archives-ouvertes.fr/hal-01021117}

Submitted on 15 Jul 2014

HAL is a multi-disciplinary open access archive for the deposit and dissemination of scientific research documents, whether they are published or not. The documents may come from teaching and research institutions in France or abroad, or from public or private research centers.
L'archive ouverte pluridisciplinaire HAL, est destinée au dépôt et à la diffusion de documents scientifiques de niveau recherche, publiés ou non, émanant des établissements d'enseignement et de recherche français ou étrangers, des laboratoires publics ou privés. 


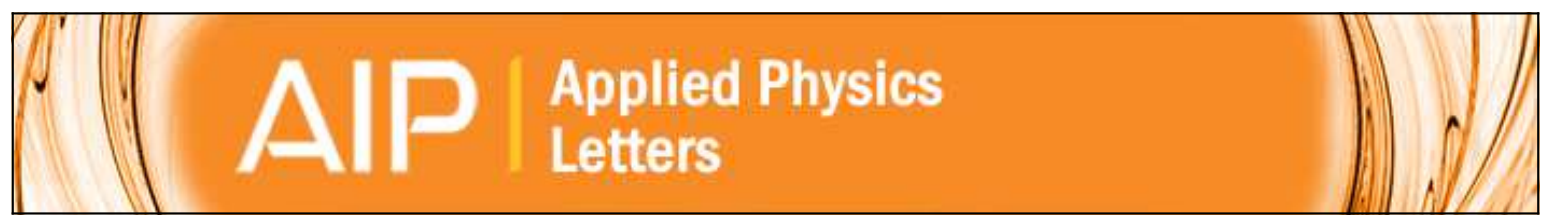

\section{Simple modeling of self-oscillations in nanoelectromechanical systems}

A. Lazarus, T. Barois, S. Perisanu, P. Poncharal, P. Manneville, E. de Langre, S. T. Purcell, P. Vincent, and A. Ayari

Citation: Applied Physics Letters 96, 193114 (2010); doi: 10.1063/1.3396191

View online: http://dx.doi.org/10.1063/1.3396191

View Table of Contents: http://scitation.aip.org/content/aip/journal/apl/96/19?ver=pdfcov

Published by the AIP Publishing

\section{Articles you may be interested in}

In-situ comprehensive calibration of a tri-port nano-electro-mechanical device

Rev. Sci. Instrum. 83, 045005 (2012); 10.1063/1.4705992

Modeling the Kelvin polarization force actuation of micro- and nanomechanical systems J. Appl. Phys. 107, 054510 (2010); 10.1063/1.3309027

Theoretical and experimental investigation of optically driven nanoelectromechanical oscillators J. Appl. Phys. 107, 034311 (2010); 10.1063/1.3305464

Self-oscillation conditions of a resonant nanoelectromechanical mass sensor J. Appl. Phys. 105, 124908 (2009); 10.1063/1.3152795

Determination of parameters for lumped parameter models of the vocal folds using a finite-element method approach

J. Acoust. Soc. Am. 106, 3620 (1999); 10.1121/1.428214

\section{A|P| Journal of}

Journal of Applied Physics is pleased to announce André Anders as its new Editor-in-Chief 


\title{
Simple modeling of self-oscillations in nanoelectromechanical systems
}

\author{
A. Lazarus, ${ }^{1}$ T. Barois, ${ }^{2}$ S. Perisanu,${ }^{2}$ P. Poncharal, ${ }^{2}$ P. Manneville, ${ }^{1}$ E. de Langre, ${ }^{1}$ \\ S. T. Purcell, ${ }^{2}$ P. Vincent, ${ }^{2}$ and A. Ayari ${ }^{2, a)}$ \\ ${ }^{1}$ Laboratoire d'Hydrodynamique, École Polytechnique, 91128 Palaiseau, France \\ ${ }^{2}$ Laboratoire de Physique de la Matière Condensée et Nanostructures, Université Lyon 1, CNRS, \\ UMR 5586, Domaine Scientifique de la Doua, F-69622 Villeurbanne Cedex, France
}

(Received 19 March 2010; accepted 25 March 2010; published online 14 May 2010)

\begin{abstract}
We present here a simple analytical model for self-oscillations in nanoelectromechanical systems. We show that a field emission self-oscillator can be described by a lumped electrical circuit and that this approach is generalizable to other electromechanical oscillator devices. The analytical model is supported by dynamical simulations where the electrostatic parameters are obtained by finite element computations. (C) 2010 American Institute of Physics. [doi:10.1063/1.3396191]
\end{abstract}

Nanoelectromechanical systems (NEMS) ${ }^{1}$ are under extensive research owing to their potential for radio frequency communication and highly sensitive sensors. This research, before becoming applicable, will have to cope with several major issues such as crosstalk. Since the work of Ref. 2, an intriguing class of NEMS has been experimentally demonstrated that could circumvent this drawback by nanoactive feedback. In contrast to quartz-oscillator like architecture, ${ }^{3}$ there is no need for macroscopic external active circuit since the nanodevice itself is placed in a self-oscillating regime. This concept was first theoretically proposed for NEMS by Gorelik et al. ${ }^{4}$ in the specific case of the charge shuttle and is now observed in a large variety of experimental configurations. $^{2,5-9}$ Although the work of Ref. 2 reaches qualitative agreement between experiment and modeling of the self-oscillation phenomenon, it lacks simple arguments about the origin of the instability. Here, we derive a simple linearized model and an equivalent purely electrical circuit that helps one getting further insight on the way to design and scale down such an oscillator. This model is then validated by dynamical and finite element simulations. The idea exposed in this article, with minor adaptations, could be useful for other experimental geometries.

In a typical experiment, a nanowire (NW) or nanotube with resistance $R_{\mathrm{NW}}$ is attached to a tungsten tip in front of an anode connected to the ground [Fig. 1(a)]. The tip is at a negative dc voltage $-V_{\mathrm{dc}}$ from the ground; electrons are emitted from the apex of the nanowire by field emission and collected by the anode. The NW starts to oscillate spontaneously in the transverse direction when $V_{\mathrm{dc}}$ is larger than some voltage threshold. This system can be modeled by two coupled differential equations [see Eqs. (1) and (2) in Ref. 2]: first, a mechanical equation that can be linearized as follows:

$$
\ddot{x}+\frac{\omega_{0}}{Q} \dot{x}+\omega_{0}^{2} x=H \bar{U} U
$$

where $x$ is the transverse displacement of the apex of the NW compared to the equilibrium position (taken positive when the NW approaches the anode), $2 \pi \omega_{0}$ the resonance frequency of the mechanical oscillator, $Q$ the quality factor, and $H$ a positive parameter characterizing the actuation strength

${ }^{a)}$ Electronic mail: anthony.ayari@lpmcn.univ-lyon1.fr. by electrostatic forces between the wire and the anode. These parameters are supposed to be relatively constant in the range of interest. $\bar{U}$ is the dc voltage between the NW and the anode and $U$ the ac voltage. $\bar{U}$ is not equal to $V_{\mathrm{dc}}$ as a result of the voltage drop through the nanowire. The linearized force is the product of $U$ and $\bar{U}$ because the electrostatic force is proportional to the square of the total voltage. Second, the linearized electrical equation reads the following:

$$
\left(\frac{\partial I_{\mathrm{FN}}}{\partial U}+\frac{1}{R_{\mathrm{NW}}}\right) U+C \dot{U}=-\frac{\partial I_{\mathrm{FN}}}{\partial x} x-C^{\prime} \bar{U} \dot{x}
$$

where $C$ is the capacitance between the NW and the anode, $C^{\prime}$ its derivative with respect to position, and $I_{\mathrm{FN}}(U+\bar{U} ; x)$ the field emission current described by the Fowler-Nordheim (FN) equation $I_{\mathrm{FN}}=A(U+\bar{U})^{2} \beta^{2} \exp [-B /(U+\bar{U}) \beta]$. The $x$ dependence of $I_{\mathrm{FN}}$ comes from the field enhancement factor $\beta$.

An important point to notice is that the field emission characteristics depends on two inputs, the apex voltage and its position, in the same way as a transistor or a vacuum tube, but the role of the gate or grid is played by the spatial degree of freedom $x$. A simple equivalent electrical circuit is shown in Fig. 1(b). The electromechanical resonator is represented
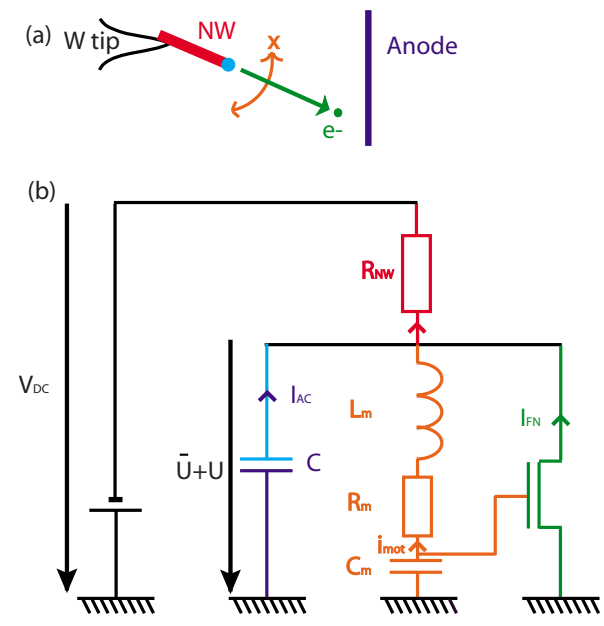

FIG. 1. (Color online) (a) Schematic of the experimental configuration and (b) schematic of the equivalent purely electrical circuit of the self-oscillation of the nanoelectromechanical system of Ref. 2. 
by a series resonant circuit in parallel with the capacitor $C$ of Eq. (2). In this well-known analogy, the motional current through the resonant circuit is $i_{\text {mot }}=C^{\prime} \bar{U} \dot{x}$ and the passive components are the motional inductance $L_{\mathrm{m}}=1 /\left(H \bar{U}^{2} C^{\prime}\right)$, the motional resistance $R_{\mathrm{m}}=\omega_{0} /\left(Q H \bar{U}^{2} C^{\prime}\right)$ and the motional capacitance $C_{\mathrm{m}}=H \bar{U}^{2} C^{\prime} / \omega_{0}^{2}$. The voltage across the motional capacitance is proportional to $x$ and can be used as the gate voltage of an equivalent transistor delivering the same field emission current for a given $x$ and $U+\bar{U}$. The transconductance of such transistor is $\left(\partial I_{\mathrm{FN}} / \partial x\right) H \bar{U} / \omega_{0}^{2}$. It brings the gain necessary to sustain the self-oscillation regime and acts as a feedback loop.

The main parameter of the self-oscillating circuit is the driving dc voltage above which the system spontaneously generates the ac signal. In the following, we derive a simple analytical formula giving the self-oscillation condition. If the nanowire resistance $R_{\mathrm{NW}}$ is smaller than the field emission resistance $\left(\partial I_{\mathrm{FN}} / \partial U\right)^{-1}$, to first order the voltage at the apex $\bar{U}$ is $V_{\mathrm{dc}}$ and there is no self-oscillation. We consider the opposite case $R_{\mathrm{NW}} \gg\left(\partial I_{\mathrm{FN}} / \partial U\right)^{-1}$ because it gives a simpler formula (the general case can be calculated straightforwardly by the same method). However, when the nanowire resistance gets larger more power is dissipated in heating instead of sustaining the oscillation, so that it might seem optimal to keep $R_{\mathrm{NW}}$ larger than the field emission resistance by less than an order of magnitude. A single differential equation of the full electromechanical system can be obtained by combining Eqs. (1) and (2), as follows:

$$
\begin{aligned}
\tau \dddot{x}+ & \ddot{x}\left(1+\frac{\omega_{0} \tau}{Q}\right)+\dot{x}\left(\frac{\omega_{0}}{Q}+H \bar{U}^{2} \tau \frac{\partial \ln C}{\partial x}+\omega_{0}^{2} \tau\right) \\
& +x\left(\omega_{0}^{2}+H \bar{U}^{2} \frac{\partial \ln \beta}{\partial x}\right)=0,
\end{aligned}
$$

where $\tau=C\left(\partial I_{\mathrm{FN}} / \partial U\right)^{-1}$ is the discharge time constant of the electrical circuit. According to the Routh-Hurwitz criterion this dynamical system is stable when the following:

$$
\begin{gathered}
H \bar{U}^{2} \tau\left[\frac{\partial \ln \beta}{\partial x}-\frac{\partial \ln C}{\partial x}\left(1+\frac{\omega_{0} \tau}{Q}\right)\right] \\
-\frac{\omega_{0} \tau}{Q}\left[\frac{1}{\tau}+\tau \omega_{0}^{2}+\frac{\omega_{0}}{Q}\right] \geq 0
\end{gathered}
$$

From this inequality, since $C$ and $\beta$ increase with $x$, only the variation in $\beta$ with $x$ favors the self-oscillation regime and we can distinguish between two categories of terms that prevent from reaching it: (i) the variation in the capacitance with $x$ and (ii) the relative value of $\tau$ and $\omega_{0}^{-1}$. The latter can be minimized for $\omega_{0} \tau \sim 1$ as long as $Q \gg 1$ (our nanowire resonators ${ }^{10}$ routinely reach $Q>10^{5}$ ). In these conditions, the geometry of the device should be such that $\partial \ln (\beta / C) / \partial x$ $>0$ to have a chance to observe self-oscillations. Finally the threshold dc voltage at the apex for self-oscillation is the following:

$$
\bar{U}_{\text {so }}=\frac{\omega_{0}}{\sqrt{Q H \partial \ln (\beta / C) / \partial x}},
$$

and the threshold dc voltage of the power supply is $V_{\text {so }}^{\mathrm{dc}}$ $=\overline{U I}_{\mathrm{so}}+R_{\mathrm{NWv}} I_{\mathrm{FN}}\left(\bar{U}_{\mathrm{so}}, \beta\right)$.

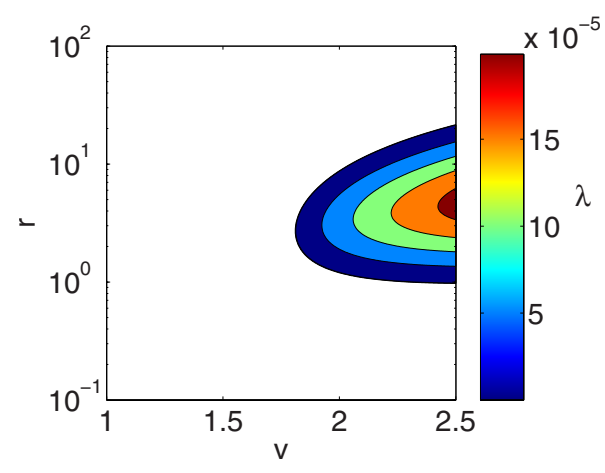

FIG. 2. (Color online) Stability map of a nanowire during field emission for $Q=10000$ and different normalized voltages $v$ and dimensionless intrinsic frequencies $r$.

In order to check the different hypotheses made, we performed numerical simulations and determined the electrostatic force, capacitance and field enhancement factor by finite element methods (FEM). The sample is a straight $10 \mu \mathrm{m}$ long nanowire of radius $100 \mathrm{~nm}$ attached to a metallic conical tip in front of a metallic plate perpendicular to the axis of the tip. The nanowire is initially tilted by $20^{\circ} \mathrm{com}-$ pared to the cone axis. The sole degree of freedom of the nanowire is this angle that can decrease due the attractive electrostatic force between the wire and the metallic plate. The distance between the tip end and the plate is $60 \mu \mathrm{m}$. The mechanical restoring force is taken from the calculated rigidity of a clamped free beam with a Young modulus of $400 \mathrm{GPa}$ and density of $3200 \mathrm{~kg} / \mathrm{m}^{3}, Q=10^{4}$ and $R_{\mathrm{NW}}$ $=10^{10} \Omega$. Further details about the simulations and a more refined mechanical model can be found in Ref. 11 . We first simulated the spatial variation in $C$ and $\beta$ and verified that $\partial \ln (\beta / C) / \partial x>0$ for a wide range of angles around $20^{\circ}$, and established that $H$ is changing by less than $15 \%$. The dimensionless differential equations were then rewritten, their eigenvalues computed, and the sign of their real part $\lambda$ scrutinized. The real part defines the growth rate of the mode and the solution, which is proportional to $\exp (\lambda t)$, decay to zero when it is negative, so that the system is stable. On the contrary, $\lambda>0$ makes the system unstable and leads it into a stable self-oscillating regime thanks to nonlinear saturating terms. The oscillation amplitude gets larger as $\lambda$ increases. Finally, we determined stability maps giving the parameter regions where $\lambda$ is positive and self-oscillations possible.

Figure 2 represents the stability map of the system for different applied dc voltages $v=V_{\mathrm{dc}} / V_{\text {ref }}$ and different dimensionless intrinsic frequencies $r=\omega_{0} \tau$. $V_{\text {ref }}=400 \mathrm{~V}$ is the voltage above which $R_{\mathrm{NW}}$ stops being negligible when compared to the field emission resistance. One can point out that (i) there is no self-oscillation for $v \ll 1$, (ii) self-oscillations are easier at higher $v$ (the growth rate is larger and the instability region wider). This validates the statement that for optimal self-oscillations $R_{\mathrm{NW}}$ needs to be bigger than the field emission resistance (the field emission current increases exponentially with $v$, so that the field emission resistance is smaller for higher $v$ ). This figure also clearly demonstrates that selfoscillations are obtained at easiest for $r \sim 1$.

We also calculated the stability map for various quality factors. Equation (5) that determines the boundary between the stable region and the self-oscillation region is in relatively good agreement with the results of numerical simula- 


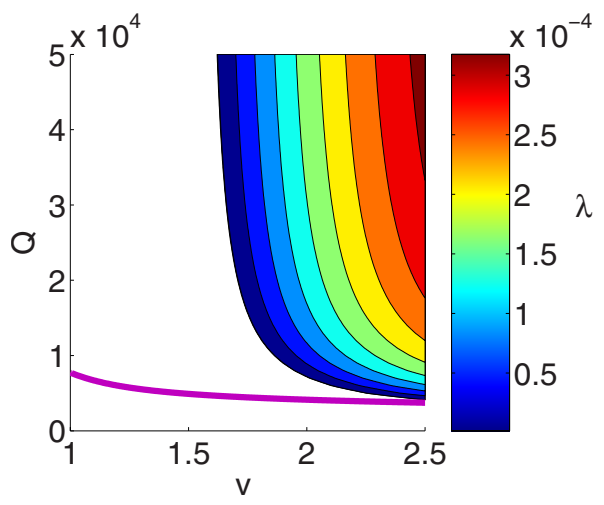

FIG. 3. (Color online) Stability map of a nanowire during field emission for a dimensionless frequency $r=5$ and different normalized voltages $v$ and $Q$. The solid line represents the self-oscillation threshold determined using Eq. (5).

tions for high voltage, i.e., when $\partial I_{\mathrm{FN}} / \partial U \gg 1 / R_{\mathrm{NW}}$. This confirms the validity of the above analytical derivation. Equation (5) shows that, as for any other NEMS device, keeping good performance (in this case by maintaining the operating voltage low) at the nanoscale and high frequency requires an improvement of the capacitive coupling and the quality factor. Finally a simple scaling calculation shows that $r$ decreases like the inverse of the apex-anode distance. Downscaling thus helps one to reach the regime where $r$ $\sim 1$. If this term become too small, or if the resistance of the nanowire or nanotube saturates in the ballistic regime, the device can still be operated with the help of an additional constant resistance between the dc power supply and $R_{\mathrm{NW}}$.

In conclusion, using an electrical equivalent circuit, we showed that the origin of self-oscillations in field emission NEMS can be understood in terms of motional capacitance and spatial variation in the field emission current in a feedback loop. An equation was derived to determine the threshold voltage for self-oscillation and its output confirmed by numerical and FEM simulations (Fig. 2 and 3). We expect that our simple model will demystify the mechanism responsible for self-oscillation in field emission NEMS, as it appears that it can be understood with simple classical electrical passive components and one transistor. It appears then that geometries like the one of Ref. 6 where the selfoscillation mechanism is not yet clearly identified are indeed very similar to ours and may be understood within the same framework. This work opens up perspectives for the control and fabrication of low power nano-oscillators for time base and ac generators applications.

This work was supported by French National Research Agency (NEXTNEMS under Project No. ANR-07-NANO008-01 and AUTONOME under Project No. ANR-07-JCJC0145-01) and Région Rhône-Alpes CIBLE program. The authors acknowledge the "plateforme nanofils et nanotubes lyonnaise."

${ }^{1}$ H. G. Craighead, Science 290, 1532 (2000).

${ }^{2}$ A. Ayari, P. Vincent, S. Perisanu, M. Choueib, M. Gouttenoire, V. Bechelany, D. Cornu, and S. Purcell, Nano Lett. 7, 2252 (2007).

${ }^{3}$ E. Colinet, L. Duraffourg, S. Labarthe, P. Andreucci, S. Hentz, and P. Robert, J. Appl. Phys. 105, 124908 (2009).

${ }^{4}$ L. Y. Gorelik, A. Isacsson, M. V. Voinova, B. Kasemo, R. I. Shekhter, and M. Jonson, Phys. Rev. Lett. 80, 4526 (1998).

${ }^{5}$ H. Kim, H. Qin, and R. H. Blick, New J. Phys. 12, 033008 (2010).

${ }^{6}$ D. Grogg, S. Ayoz, and A. Ionescu, Proceedings of the International Electron Devices Meeting (IEDM), 2009 (unpublished), p. 793.

${ }^{7}$ V. I. Kleshch, A. N. Obraztsov, and E. D. Obraztsova, JETP Lett. 90, 464 (2009)

${ }^{8}$ G. A. Steele, A. K. Huttel, B. Witkamp, M. Poot, H. B. Meerwaldt, L. P. Kouwenhoven, and H. S. J. van der Zant, Science 325, 1103 (2009).

${ }^{9}$ K. L. Phan, P. G. Steeneken, M. J. Goossens, G. E. J. Koops, G. J. A. M. Verheijden, and J. T. M. van Beek, arXiv:0904.3748v1 (unpublished).

${ }^{10}$ S. Perisanu, P. Vincent, A. Ayari, M. Choueib, S. Purcell, M. Bechelany, and D. Cornu, Appl. Phys. Lett. 90, 043113 (2007).

${ }^{11}$ A. Lazarus, E. de Langre, P. Manneville, P. Vincent, S. Perisanu, A. Ayari, and S. Purcell, "Statics and dynamics of a nanowire in field emission,"Int. J. Mech. Sci. (to be published). 